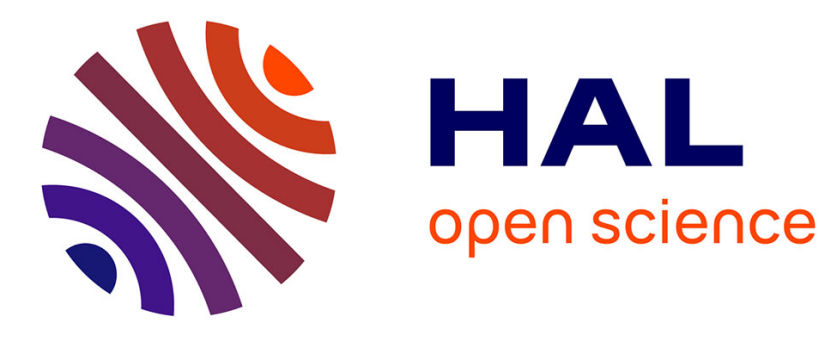

\title{
Current-conductance and stress-elastic modulus correlations
}

Stéphane G. Roux, François Hild, Didier Fokwa, Denis Breysse, Giusepe

Geymonat, Gilles Pijaudier-Cabot

\section{To cite this version:}

Stéphane G. Roux, François Hild, Didier Fokwa, Denis Breysse, Giusepe Geymonat, et al.. Currentconductance and stress-elastic modulus correlations. Journal de Physique II, 1991, 1 (3), pp.265-272. 10.1051/jp2:1991167 . jpa-00247515

\section{HAL Id: jpa-00247515 https://hal.science/jpa-00247515}

Submitted on 1 Jan 1991

HAL is a multi-disciplinary open access archive for the deposit and dissemination of scientific research documents, whether they are published or not. The documents may come from teaching and research institutions in France or abroad, or from public or private research centers.
L'archive ouverte pluridisciplinaire HAL, est destinée au dépôt et à la diffusion de documents scientifiques de niveau recherche, publiés ou non, émanant des établissements d'enseignement et de recherche français ou étrangers, des laboratoires publics ou privés. 
Classification

Physics Abstracts

$62.20-62.20 \mathrm{M}-02.60$

\title{
Short Communication
}

\section{Current-conductance and stress-elastic modulus correlations}

\author{
Stéphane Roux $\left({ }^{1, *}\right)$, François Hild $\left({ }^{2}\right)$, Didier Fokwa $\left({ }^{2}\right)$, Denis Breysse $\left({ }^{2}\right)$, Giusepe Gey- \\ monat $\left({ }^{2}\right)$ and Gilles Pijaudier-Cabot $\left({ }^{2}\right)$
}

(1) Centre d'Enseıgnement et de Recherche en Analyse des Matérıux, Ecole Natıonale des Ponts et Chaussées, 1 Avenue Montaigne, Central IV, F-93167 Norsy le Grand Cedex, France

$\left(^{2}\right)$ Laboratorre de Mécanıque et Technologıe( $\left.{ }^{* *}\right)$, Ecole Normale Supérıeure de Cachan, Unıversıté Parıs VI, 61 Avenue du Présıdent Wilson, F-94235 Cachan Cedex, France

(Recetved 5 December 1990, accepted 17 January 1991)

\begin{abstract}
We consider a composite medium which consists in resistance elements with a distribution of conductance, in a narrow range around a mean value. The question we address is the distribution of local power dissipation, and its correlation with the local conductance. Various discretizations of the problem are considered: regular networks of different types in two and three dimensions whose bonds are assigned conductances at random, finite element method with random distribution of conductances in each element. We also consider the case of elastic elements, and study the distribution of elastic energy stored in each element. It is shown that the correlation between the dissipated (or elastic) energy and the conductance (or elastic modulus) depends on the discretization. These correlations are analysed in an effective medium theory framework, and numerical simulations confirm the theoretical predictions. The distribution of local energy always tends towards a Gaussian distribution for all cases considered, in the limit of a small disorder.
\end{abstract}

\section{Introduction.}

Heterogeneous materials occur extremely frequently in nature. However, the influence of the heterogeneity on vårious physical properties can vary a lot. In particular, a weak disorder (narrow distribution of local properties) will have a very limited impact on the overall transport properties of the system. However, when considering brittle fracture or damage, even a vanishing disorder may controll the macroscopic fracture properties, and thus it is extremely important to know more about the local state of stress of an heterogeneous elastic brittle solid in order to understand the initiation and development of damage and fracture. The purpose of this paper is to analyse the distribution of local stress, and to study the correlations between stress and local elastic properties. We will mainly focus on a comparable case, e.g. electrical properties, since all results can be straightforwardly extended to elasticity at the cost of more cumbersome calculations.

(*) Also at LPMMH, ESPCI, 10 rue Vauquelın, F-75231 Parıs Cedex 05, France.

$\left({ }^{* *}\right)$ URA CNRS 860 
The reported results are relevant for a numerical approach to brittle fracture of monolithic ceramics [1]. Indeed in such cases, initial defects control the failure, and thus it suffices to compute the stress distribution using linear elasticity to be able to predict the breaking of the first element in the material, which corresponds to the failure of the whole material (weakest link hypothesis). In addition, some statistical information is available for the defect distribution, and thus our approach allows to extend a previous approach [1] coupling the defect distribution to the fracture properties without having to resort to numerical computations.

\section{Effective medium approximation for electrical networks.}

Let us consider a regular network whose bonds $i$ are assigned a conductance $g_{i}$ randomly picked from a distribution $p(g)$. The question we address in this paper is the distribution of local power dissipation $e=j^{2} / g$ (where $j$ is the current flowing through a bond), and the eventual correlations between $e$ and $g$. In particular, we will be interested in the limit where the distribution of conductance is narrow, i.e. a small disorder case.

In the case of a small disorder, the Effective Medium Approximation (hereafter referred to as EMA) is known to give an extremely accurate determination of the conductivity. We will show that it also allows to predict the correlation between $e$ and $g$ locally. We will follow closely the derivation of the EMA result given by Kirkpatrick [2] for random resistor networks. The EMA is obviously applicable to much more complex cases, and in particular for elasticity, we refer to [3] for a more general presentation.

The spirit of the EMA method is to focus on one particular element, and consider that the rest of the medium can be well approximated by a homogeneous medium, i.e. in this case, a regular network composed of identical resistors. We follow this approximation, and consider an infinite homogeneous network, whose bonds are all assigned the same conductance, $g$, apart from one bond, AB, whose conductance is called $h$, see figure 1 . A preliminary question we need to answer first is to know what is the current flowing through the bond $A B$, when a uniform mean current is imposed at infinity, in such a way that the current flowing in bond $\mathrm{AB}$ would be $j_{0}$ if $h=g$.

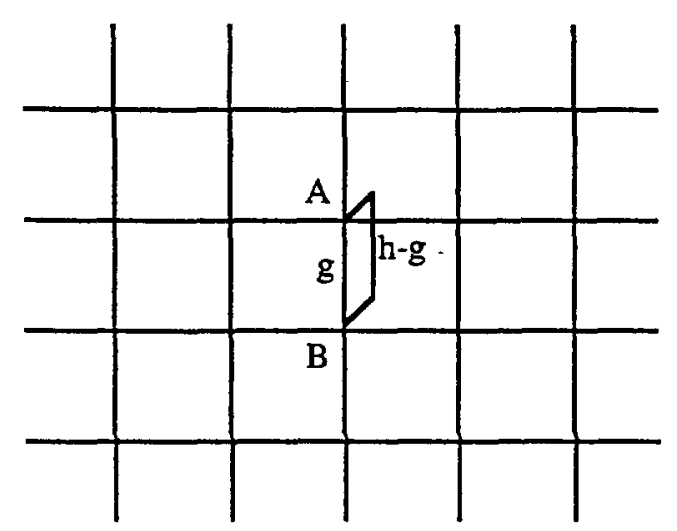

Fig. 1. - Schematic decomposition of the network into two parts: one homogeneous lattice where all bonds have the same conductance $g$ and one additional conductance $h-g$ placed in parallel with the bond AB. This construction is equivalent to the case of one bond $\mathrm{AB}$ having a conductance $h$ embedded in a homogeneous lattice. 
To answer this question, we split the bond $A B$ into two elements mounted in parallel, one with a conductance $g$, and one with a concuctance $h-g$. Formally, this partition is only possible when $h>g$, however, an algebraic continuation to negative $h-g$ makes no difference. From this construction, we see that the network can be seen as a homogeneous one, and an additional conductance $h-g$ has been placed in parallel to the bond AB. We can now use a superposition of two different boundary conditions imposed to the homogeneous network. The first one simply consists in applying the boundary condition chosen previously at infinity. By construction, the current flowing in the bond $\mathrm{AB}$ is simply $j_{1}=j_{0}$. The voltage drop accross it is thus $v_{1}=j_{0} / g$. The second situation consists in sending a current $k$ in $\mathrm{A}$ and collecting it in $\mathrm{B}$, whereas no current is sent at infinity. Let us call $G$ the conductance of the infinite homogeneous lattice when a current is sent in $A$ and collected in $B$. The voltage drop accross $A B$ in the second case will be $v_{2}=$ $k / G$, whereas the current flowing through the bond $\mathrm{AB}$ in the lattice will be $\jmath_{2}=(g / G) k$ We now superpose both cases to solve our problem. The current $k$ is determined by noting that it is the one which flows through the parallel bond of conductance $h-g$, and thus which satisfies $(h-g)\left(v_{1}+v_{2}\right)=-k$ Solving this equation for $k$ gives

$$
k=-\frac{(h-g) G}{g(G+h-g)}
$$

Obviously, $G$ is proportional to $g$ The proportionality constant $A=G / g$ is a characteristic of the lattice. Since we will mainly consider a small disorder limit, we introduce $\varepsilon$ such that $h=g(1+\varepsilon)$. Using these notations, $k$ reads

$$
k=\frac{-A \varepsilon}{(A+\varepsilon)} \jmath_{0}
$$

Coming back to our initial problem, the total current flowing through the bond $A B$ of conductance $h$ is

$$
\jmath_{\mathrm{AB}}=j_{1}+j_{2}=\frac{A(1+\varepsilon)}{(A+\varepsilon)} \jmath_{0}
$$

The energy dissipation in the bond $\mathrm{AB}, e_{\mathrm{AB}}=j_{\mathrm{AB}}^{2} / h$ can be expanded to the second order in $\varepsilon$ to give

$$
e_{\mathrm{AB}}=\left(e_{0}\right)\left\{1+\varepsilon(1-2 / A)+\varepsilon^{2}\left(3 / A^{2}-2 / A\right)+O\left(\varepsilon^{3}\right)\right\}
$$

where we have introduced $e_{0}=j_{0}^{2} / g$, the dissipation for $h=g$, in order to make the result not-dimensional.

We finally have to find the value of $A$ in order to conclude. We refer to [2], for an elegant solution to this question. The answer is $A=z / 2$ where $z$ is the coordinance of the lattice (the coordinance is the number of bonds which are connected to a node). For the square lattice, $z=4$ and thus $A=2$. We see from equation (4) that the correlation between $e_{\mathrm{AB}}$ and $g_{\mathrm{AB}}=h$ is only to the second order. A linear regression of $e$ versus $h$ will give no correlation. Up to the second order we can write

$$
\frac{e-e_{0}}{e_{0}}=\tilde{(-1 / 4)}\left(\frac{h-g}{g}\right)^{2}+O\left(\left(\frac{h-g}{g}\right)^{3}\right)
$$

In fact, for the special case of the square lattice, we can use a duality transformation [4] to prove that the first order term in $(h-g)$ is zero in this relation.

For a triangular lattice, $z=6$, the linear term in equation (4) does not cancel out. It gives:

$$
\frac{e-e_{0}}{e_{0}}=(1 / 3)\left(\frac{h-g}{g}\right)-(1 / 3)\left(\frac{h-g}{g}\right)^{2}+O\left(\left(\frac{h-g}{g}\right)^{3}\right)
$$


Thus, the smaller the conductance, the larger the dissipation. For a honeycomb lattice, $z=3$, the trend is opposite:

$$
\frac{e-e_{0}}{e_{0}}=-(1 / 3)\left(\frac{h-g}{g}\right)+O\left(\left(\frac{h-g}{g}\right)^{3}\right) .
$$

This conclusion is a little surprising since, depending on the details of the discretization, a general argument cannot state where the maximum dissipation is more likely to occur: for good or bad conductors. This observation should call the attention onto models of electrical or mechanical damage where depending on the details of the discretization, a damaging process can either be stabilized or destabilized, according to the fact that further damage is respectively less or more likely to occur in regions of space where some damage is already present.

\section{Numerical simulations.}

We have checked numerically these predictions by solving the current distribution in lattices where the conductance of the bonds were randomly sampled over a uniform distribution $[1-\delta / 2,1+\delta / 2]$. We tested different lattices: square and triangular lattices in two dimensions and a centered cubic in three dimensions. The sizes of the lattices investigated were $20 \times 20$ in two dimensions, and $20 \times 20 \times 20$ in three dimensions. We chose a width $\delta=1 \%$. The currents were obtained using a conjugate gradient relaxation routine with a precision of $10^{-10}$. In all cases, we performed a linear regression of the dissipated energy versus the conductance for all bonds in the network. We thus had access to the contribution which is linear in $\varepsilon$ in equation (4). We obtained the following estimates of the slopes: $0.0,0.35$ and 0.52 for respectively the square, the triangular and the $c c$

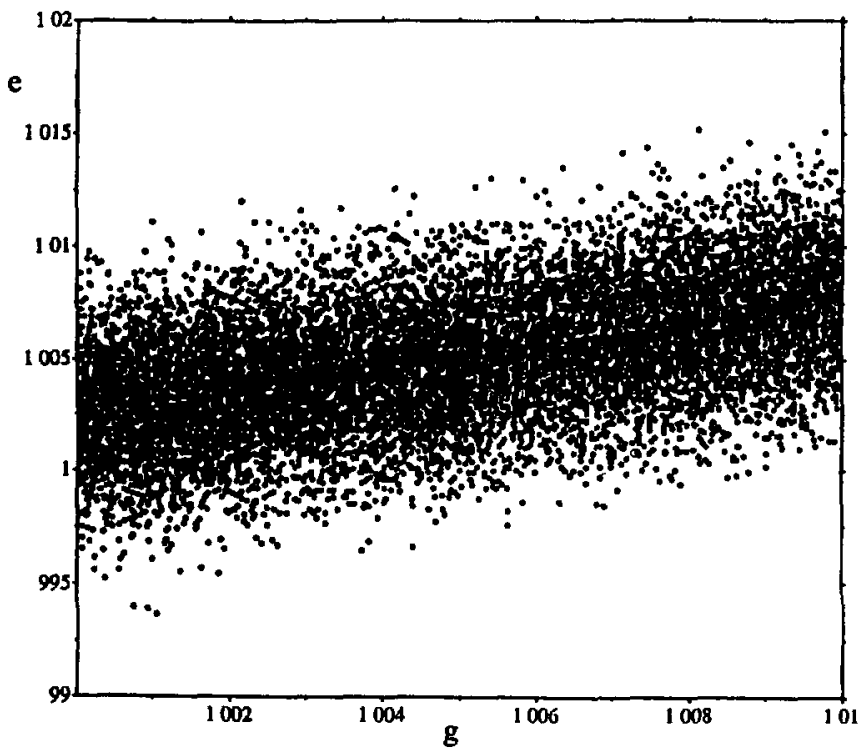

Fig. 2. Distribution of the local energy dissipated $e$ in a bond of conductance $g$, in a three dimensional c.c. lattice of size 15 . One sees a mean linear correlation between $e$ and $g$ as well as a statistical distribution of $e$ roughly independent from $g$. 
lattice. These estimates should be compared to the expected values $0,1 / 3$, and $1 / 2$ (using Eq.(4) with $z=4,6$ and 8 respectively). Figure 2 shows an illustrative example of the cc lattice for a size 15 (13500 bonds).

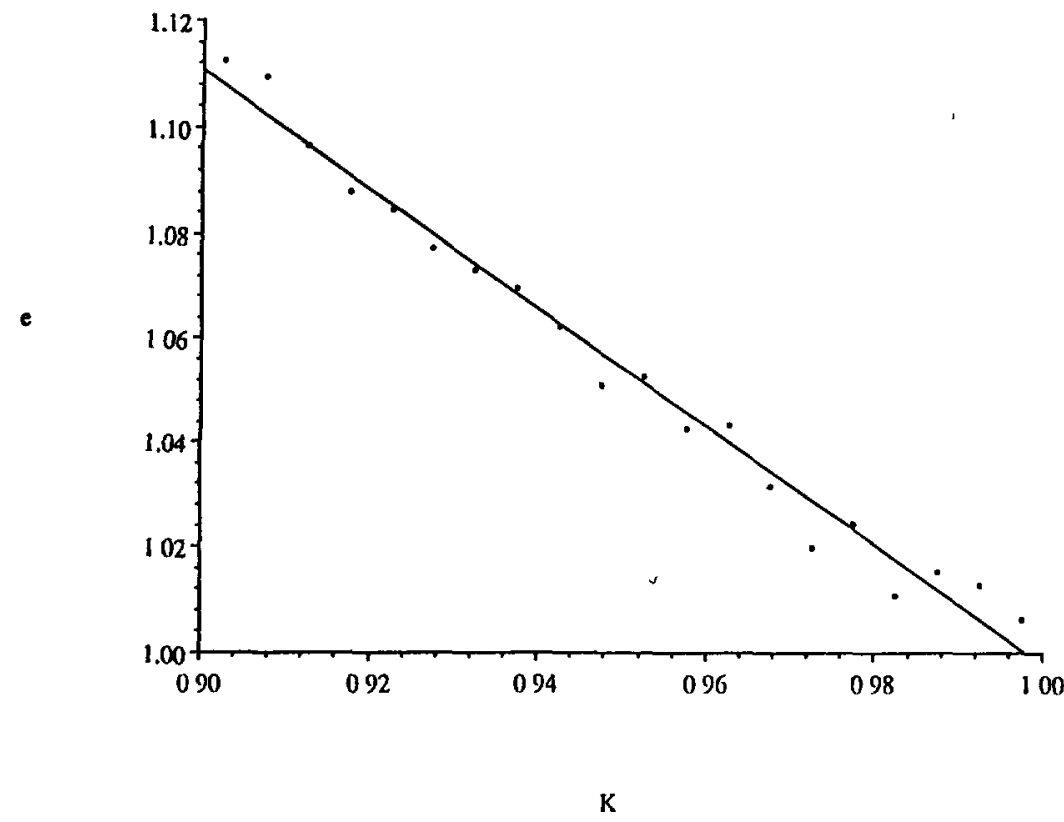

Fig. 3. - Average elastıc energy $e$ stored in cells having an elastic modulus $K$ The data were obtained using a two dimensional finite element code.

We have also tested this correlation for a hierarchical diamond model, where the topology of the lattice is different from that of a Euclidian lattice. We expect however the same result as equation (4) in such a case. The value of $A$ can be computed easily in this case, and amounts to $3 / 2$ (the relation between $A$ and $z$ does not hold in this case). We measured a slope -0.33 , in agreement with the expected result $-1 / 3$.

Is it possible to relate this slope to another characteristic of the network that may be measured globally, and not locally as presented up to now? We propose to use a EMA result to do this. Let us imagine that we start with a homogeneous network, and remove at random a finite fraction of bonds $q$. The removal of these bonds will reduce the global conductivity of the network by an amount which is proportional to $q$ for $q \ll 1$. The EMA prediction is

$$
\frac{G(q)}{G(q=0)}=1-\lambda q
$$

where $\lambda=(A-1) / A$. The decrease of the total conductance is a quantity which can easily be measured. Eliminating $A$ between equations (4) and (8), we obtain a first order correlation coefficient $(1-2 A)=2 \lambda-1$.

Up to now we have simply dealt with regular lattices. What can be said about the finite element method as an alternative discretization of the heterogeneous medium? Assuming that each element is given an independent conductivity, and that the elements are of first order, we see that the discretized problem can readily be mapped onto a resistor network. The result equation 
(4) is thus expected to hold, provided the factor $A$ is computed in the corresponding framework, i.e. one has to solve the problem of a source located in one element (far from the border) in an otherwise homogeneous medium.

For linear elasticity, we can reproduce a very similar argument, although all scalar quantities (such as $A, \varepsilon, \quad$ ) have to be turned into tensors. To illustrate the validity of the results, we have performed some simple simulations using linear elasticity, with a finite element code in two dimensions. The elastic modulus was chosen uniformly distributed between 0.9 and 1.0. Figure 3 shows the average of the elastic energy, $e$, stored in cells having an elastic modulus $K$. The data are averaged over 25 -samples of size 10 . We see that a linear correlation accounts very well for the presented results.

\section{Statistical distribution of local energy.}

We propose now to go a little further in the analysis of the current distribution in the medium. indeed from figure 2, we see that despite the correlation between the local energy and the conductance, the former displays a statistical distribution which hides partially the mean correlation. what is the distribution of local current in the medium?

From the above proposed derivation, we see that the spreading of local current comes from the fluctuations of resistance of the network seen from one bond (fluctuation of $A$ ). These fluctuations are obviously independent of the properties of the bonds, and thus justifies that the distribution of local energy appears to be independent of the local conductance as may be seen from figure 2 . To derive this distribution, we will start with a homogeneous network, all bonds having the same conductance $g$ and consider the change of $A$ that will result from the change of the conductance of any bond $i$ in the network. The fluctuation of $A$ will then be the sum over all bonds of these variations. the spirit of this approach is similar to that of the EMA since the influence of one bond on the conductance of the whole network seen from another bond is computed using a fictituous homogeneous network.

A variation $\delta A$ in $A$ will induce a change $\delta j_{\mathrm{AB}}$ in the current $j \mathrm{AB}$. The relation between these two variations is given by the derivative of equation (3):

$$
\delta j_{\mathrm{AB}}=-\frac{\varepsilon(1+\varepsilon)}{(A+\varepsilon)^{2}} \delta A
$$

To compute the change in $A$ which result from a variation $\delta g_{z}$ of the conductance of a bond $z$ different from $\mathrm{AB}$, we use Cohn's theorem. We imagine applying a voltage drop $U_{\mathrm{AB}}$ across the bond $\mathrm{AB}$. The voltage difference on the bond $\imath$ will be $u_{2}$. Cohn's theorem simply states that

$$
\delta A_{\imath}=\frac{\delta g_{i}}{g} \frac{u_{i}^{2}}{U_{\mathrm{AB}}^{2}}
$$

The voltage drop $u_{z}$ is simply a dipole field which decays with the distance $x$ from AB to $i$ as $x^{-d}$ We now have all the ingredients necessary to conclude.

The total variation in current $\delta_{\mathrm{AB}}$ is the sum over all bonds $i$ of each contribution to $\delta A$.

$$
\delta j_{\mathrm{AB}}=-\frac{\varepsilon(1+\varepsilon)}{(A+\varepsilon)^{2} g U_{\mathrm{AB}}^{2}} \sum_{\imath} \delta g_{3} u_{3}^{2}
$$

Thus the fluctuation of $\jmath_{\mathrm{AB}}$ appears to a simple sum of decorrelated numbers having different werghts $\left(u_{z}^{2}\right)$. The mean value of $\delta \jmath_{\mathrm{AB}}$ is zero since the average of $\delta g_{z}$ is zero. The width of the 
distribution of $\delta j_{\mathrm{AB}}$ can be estimated using the variance of this quantity. Calling $\sigma(g)^{2}$ the variance of the distribution of $g$, we can compute

$$
\left\langle\delta j_{\mathrm{AB}}^{2}\right\rangle=\left(\frac{\varepsilon(1+\varepsilon)}{(A+\varepsilon)^{2} g U_{\mathrm{AB}}^{2}}\right)^{2} \sum_{z} \sigma(g)^{2} u_{\imath}^{2}
$$

Using the dependence of $u_{i}$ with the distance $x$, it is obvious that the sums in equation (11-12) are convergent. It also shows that most of the fluctuations in $j$ are due to the local environment. We underline in this last result, that the width of the distribution of current at fixed $\varepsilon$ is proportional to the width of the distribution in conductance.

In the case where the local conductances are distributed normally, then the distribution of $\jmath$ is also along a Gaussian since we deal with a sum of independent variable, albeit with different weights. The fact that the sums are convergent prevents us from applying the central limit theorem in the case of any non-Gaussian distribution of $g$ : the distribution of $\jmath$ will not converge towards a Gaussian as the system size increases to infinity. Our situation ss close to that of a sum of a finite number of uncorrelated random numbers. In practice, provided the distribution of $g$ is narrow, that of $j$ will be very close to a Gaussian. For the local energy distribution, we have to take the convolution product of the distributions of $j$ and $g$ with weights 2 and 1 respectively. Again this distribution will be close to a Gaussian in a small disorder lımit.

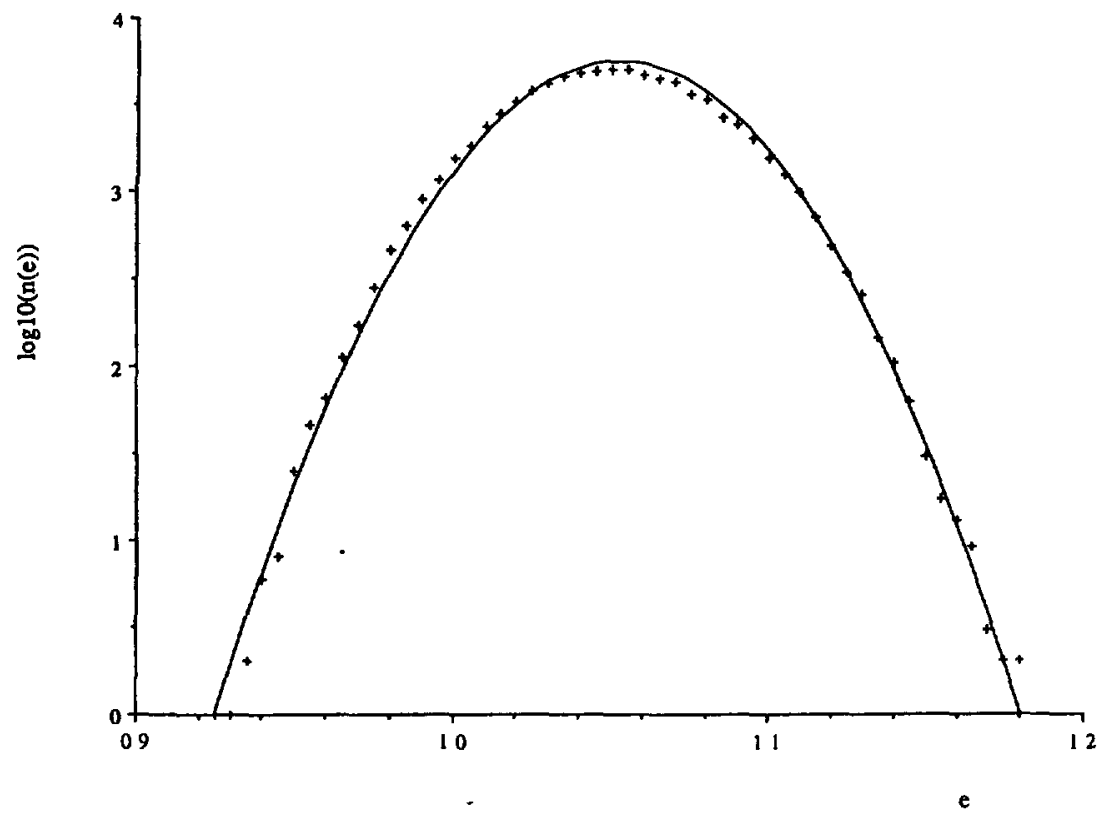

Fig. 4. - Semı-log histogram of the distribution of local energy dissipated in square lattıces $(+)$. A parabolic fit is shown as a full curve for comparison to a Gaussian distribution.

Figure 4 shows the semi-log plot of the histogram of the local energy dissipated in $20 \times 20$ twodimensional square lattıces with a uniform distribution of local conductances of width $\delta g / g=1 \%$ One sees that this plot is very close to a parabola, and thus the distribution is very close to a Gaussian. We have also verified that the width of the distribution of $e$ is proportional to that of $g$, when the disorder is small. 
One can show that the distribution of conductance of finite size lattices converges toward a normal distribution when the size tends to infinity. Thus the previous results allow to state a more general property: Under coarse graining, the distribution of current in an heterogeneous conductor will tend toward a Gaussian distribution. In addition both distribution will have a similar width, and thus the distribution of current coarse-grained at a scale $\ell$ has a width which decreases as $\ell^{-d / 2}$.

\section{Conclusion.}

Let us stress finally that all the results reported above are valid as long as the distributions of conductances.or elastic moduli are narrow. In practice however, for the elastic case, we tested a distribution of damage extending from 0 to 0.5 without noticing any strong deviation from the linear correlation between energy and elastic modulus. Let us note that in the case of an extremely broad distribution of conductances or elastic moduli, it is possible to use a very different approach, [5] and it can be found that the local energy distribution can be characterized by a generalized multifractal form, which take into account both the size effect and the disorder dependence.

Let us stress finally the strong discretization dependence of the correlations between the local transport properties and the local energy distribution. This puzzling result shows that in the continuum limit, one has to precise some informations on the shapes of the different elements which are given a constant transport property. Otherwise the result is ill-posed. Applications of the results presented above to the case of fatigue are currently being investigated.

\section{References}

[1] Hild F., AMAR E. and MARQUis D., in Proceedıng of MECAMAT Meetıng (Senlıs, France) 10/5/90.

[2] KIRKPATRICK S , Rev. Mod. Phys. 45 (1973) 574

[3] SANCHEZ-PALENCIA E and ZAOUi A. Eds., Homogenization techniques for composite media (Proceedings Udine, Italy 1985) Lecture notes in Physics (Springer) 1987.

[4] STRALEY J.P, Phys. Rev B 15 (1977) 5733.

[5] RouX S , Rigord P., HANSEN A. and HiNRICHSEN E L., "Power dissipation in a random resistor network having a broad distributıon of conductıvitıes", preprint.

Cet artıcle a été ımprımé avec le Macro Package "Edıtıons de Physıque Avrıl 1990" 